\title{
REMARK ON FREE PRODUCTS OF GROUPS $\left({ }^{1}\right)$
}

\author{
BY \\ P. J. HILTON
}

1. Introduction. At the Louvain Colloquium in 1956, G. W. Whitehead raised the question of finding, in some sense, a dual of Lusternik-Schnirelmann category for topological spaces (see [1]); that is, a numerical invariant $k$ to be attached to a space such that the spaces with $k \leqq 2$ are just those admitting multiplications. It has appeared convenient in fact to seek such an invariant in the category of c.s.s. complexes by utilizing Kan's loop-functor $G$ (see $[2 ; 3]$ ) which passes to the category of group complexes. This has required an investigation of certain properties of free products of groups which it seemed worthwhile extracting from the general body of the investigation and presented separately. It is hoped to publish the topological applications later; in this note we are content at the end to indicate briefly what these are.

The author wishes to acknowledge that the Cartesian product representation of the free product, given by Theorem 2.20, was first suggested to him by John Milnor at the 1956 Mexico Symposium.

2. The natural factorization. Let $G_{1}, G_{2}, \cdots, G_{n}$ be groups, let $*_{i} G_{i}$ $=G_{1} * \cdots * G_{n}$ be their free product, let $\times_{i} G_{i}=G_{1} \times \cdots \times G_{n}$ be their direct product and let

$$
\kappa: *_{i} G_{i} \rightarrow \times_{i} G_{i}
$$

be the natural map from free product to direct product. Then $\kappa$ is an epimorphism and we proceed now to describe a canonical factorization of $\kappa$.

If $I$ is an ordered subset of the ordered set $\{1,2, \cdots, n\}$ and has $r$ members $i_{1}, \cdots, i_{r}$ we write $|I|=r$ and call $I$ an $r$-string; we also write $G_{I}$ for $G_{i_{1}} * \cdots * G_{i_{r}}$. If $I$ is empty $G_{I}$ is the trivial group. If $J \subseteq I$ there is a projection $\pi_{J}^{I}: G_{I} \rightarrow G_{J}$, and we consider the inverse system $S_{q}=\left(G_{I}, \pi_{J}^{I}\right)$ where $I$ ranges over all strings with $|I| \leqq q$. An element $g$ of the limit group $G^{a}$ is then a collection of elements $\left\{g_{I}\right\},|I| \leqq q$, such that $\pi_{J}^{I} g_{I}=g_{J}$. The group $G^{q}$ may be identified in a natural way with a subgroup of $X_{|I|=q} G_{I}$ where $\left\{g_{I}\right\}$, $|I|=q$, as in the subgroup if and only if

$$
\stackrel{I}{\pi_{I} \cap I^{\prime}} g_{I}=\pi_{I^{\prime} \cap^{\prime}}^{I^{\prime}} g_{I^{\prime}}
$$

for each pair of $q$-strings $I, I^{\prime}$. The identification is of course achieved by taking an element $\left\{g_{I}\right\}$ of $G^{q}$ and retaining those components $g_{I}$ for which

Received by the editors November 6, 1959.

(1) Research sponsored by the Office of Naval Research under Contract No. Nonr 401(20)NR 043-167. 
$|I|=q$. It is worth remarking that it is sufficient to demand (2.1) for $q$ strings $I, I^{\prime}$ which are adjacent in the sense that $I \cap I^{\prime}$ is a $(q-1)$-string. For given any two $q$-strings $I, I^{\prime}$ we may find a chain of $q$-strings $I_{0}, I_{1}, \cdots, I_{k}$ such that $I_{0}=I, I_{k}=I^{\prime}, I_{j}$ and $I_{j+1}$ are adjacent and $I_{j} \cap I_{j+1} \supseteq I \cap I^{\prime}, j=0$, $1, \cdots, k-1$.

There is a canonical homomorphism $\kappa^{q}: G^{q+1} \rightarrow G^{q}$, given by

$$
\kappa^{q}\left\{g_{I}\right\}=\left\{g_{J}\right\},
$$

where $I$ runs over all strings with $|I| \leqq q+1$ and $J$ runs over all strings with $|J| \leqq q$.

Notice that (after identification) $*_{i} G_{i}=G^{n}$ and $\times_{i} G_{i}=G^{1}$. We then have

Proposition 2.3. $\kappa=\kappa^{1} \circ \kappa^{2} \circ \cdots \circ \kappa^{n-1}$.

Now let $\phi_{i}: G_{i} \rightarrow H_{i}, i=1, \cdots, n$, be a set of homomorphisms. It is clear that the maps $\phi_{i}$ induce homomorphisms $\phi^{q}: G^{q} \rightarrow H^{q}$ such that

$$
\phi^{q} \kappa^{q}=\kappa^{q} \phi^{q+1}, \quad q=1, \cdots, n-1 .
$$

THEOREM 2.5. There is a transformation $\eta^{q}: G^{q} \rightarrow G^{q+1}$ such that $\kappa^{q} \eta^{q}=1$ and $\eta^{q}$ is natural in the sense that

$$
\phi^{q+1} \eta^{q}=\eta^{q} \phi^{q} .
$$

Notice that it is not asserted that $\eta^{q}$ is a homomorphism. We now prove the theorem. Let $\lambda^{q}=\kappa^{q} \circ \cdots \circ \kappa^{n-1}: G^{n} \rightarrow G^{q}$. We filter $G^{q}$ by declaring $g=\left\{g_{I}\right\} \in F_{s}\left(G^{q}\right), 0 \leqq s \leqq q$, if $\left({ }^{2}\right) g_{I}=e$ whenever $|I|=s$. Then clearly

$$
(e)=F_{q}\left(G^{q}\right) \subseteq \cdots \subseteq F_{o+1}\left(G^{q}\right) \subseteq F_{s}\left(G^{q}\right) \cdots \subseteq F_{0}\left(G^{q}\right)=G^{q} .
$$

We now define transformations $\alpha_{s}: F_{s} \rightarrow F_{s+1}, \beta_{s}: F_{s} \rightarrow G^{n}$ so that

$$
g=\alpha_{s}(g) \lambda^{a} \beta_{s}(g), g \in F_{s}, \quad 0 \leqq s \leqq q-1 .
$$

We first order the $r$-strings for each $r, 0 \leqq r \leqq n$; we then set $\alpha_{s}(g)=g^{\prime}$ where

$$
g_{I}^{\prime}=g_{I}\left(\underset{|J|=s+1 ; J \subseteq I}{\text { product }} g_{J}\right)^{-1}
$$

here we regard $G_{J}$ as embedded in $G_{I}$ if $J \subseteq I$. Further we set $\beta_{s}(g)=g^{\prime \prime}$ where

$$
g^{\prime \prime}=\underset{|J|=s+1}{\operatorname{product}} g_{J}
$$

here we regard $G_{J}$ as embedded in $G^{n}=*_{i} G_{i}$.

We have to verify that $g^{\prime} \in F_{s+1}\left(G^{q}\right)$ and that (2.7) holds. The critical observation is that, since $g \in F_{s}$,

$$
\stackrel{I}{\pi_{K}} g_{J}=e \text { if } J \nsubseteq K,
$$

(2) The symbol " $e$ " represents the identity element of any group. 
while

$$
\stackrel{I}{\pi_{K}} g_{J}=g_{J} \text { if } J \subseteq K,
$$

where $|J|=s+1$. It follows immediately that $g^{\prime} \in G^{q}$. For $\pi_{K}^{l} g_{I}^{\prime}$ $=\pi_{K}^{I} g_{I}\left(\text { product }_{|J|-s+1 ; J \subseteq I} \pi_{K}^{I} g_{J}\right)^{-1}=g_{K}\left(\text { product }_{|J|-s+1 ; J} \subseteq_{K} g_{J}\right)^{-1}$, by $(2.10)$, $=g_{K}^{\prime}$. Trivially $g^{\prime} \in F_{s+1}\left(G^{a}\right)$; for, if $|I|=s+1, g_{I}^{\prime}=g_{I} g_{I}^{-1}=e$. Now let us write $\pi_{I}$ for $\pi_{I}\{1, \ldots, n\}$; then

$$
\left(\alpha_{s}(g) \lambda^{a} \beta_{s}(g)\right)_{I}=g_{I}^{\prime} \pi_{I} g^{\prime \prime}=g_{I}\left(\underset{|J|=s+1 ; J_{I} \subseteq_{I} I}{\operatorname{product}} g_{J}\right)^{-1}\left(\underset{|J|=s+1}{\operatorname{product}} \pi_{I} g_{J}\right) .
$$

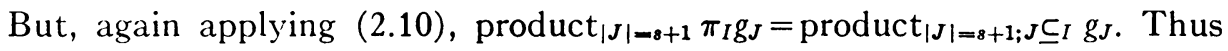

$$
\left(\alpha_{s}(g) \lambda^{a} \beta_{s}(g)\right)_{I}=g_{I}
$$

and (2.7) is proved. It is moreover plain that the maps $\alpha_{s}, \beta_{s}$ have the naturality properties

$$
\phi^{q} \alpha_{s}=\alpha_{s} \phi^{q}, \quad \phi^{n} \beta_{s}=\beta_{s} \phi^{q} .
$$

Set $\theta_{0}=1, \theta_{s}=\alpha_{s-1} \theta_{s-1}: G^{q} \rightarrow F_{s}\left(G^{q}\right), 1 \leqq s \leqq q-1$, and let

$$
\tau^{q}: G^{q} \rightarrow G^{n}
$$

be given by

$$
\tau^{q}=\prod_{s=q-1}^{0} \beta_{s} \theta_{s}
$$

thus explicitly, if $g \in G^{q}, \tau^{q}(g)=\beta_{q-1} \theta_{q-1}(g) \cdots \beta_{1} \theta_{1}(g) \beta_{0} \theta_{0}(g)$. An easy calculation based on (2.7) shows that

$$
\lambda^{q} \tau^{q}=1
$$

moreover the relations (2.11) imply that

$$
\phi^{n} \tau^{q}=\tau^{q} \phi^{q}:
$$

for, arguing by induction, we find $\phi^{a} \theta_{s}=\theta_{s} \phi^{q}$ whence

$$
\phi^{n} \tau^{q}=\prod_{s=q-1}^{0} \phi^{n} \beta_{s} \theta_{s}=\prod_{s=q-1}^{0} \beta_{s} \phi^{q} \theta_{s}=\prod_{s=q-1}^{0} \beta_{s} \theta_{s} \phi^{q}=\tau^{q} \phi^{q} .
$$

Finally we define $\eta^{q}: G^{q} \rightarrow G^{q+1}$ by

$$
\eta^{q}=\lambda^{q+1} \tau^{q} .
$$

Then $\kappa^{q} \eta^{q}=\kappa^{q} \lambda^{q+1} \tau^{q}=\lambda^{q} \tau^{q}=1$ and $\phi^{q+1} \eta^{q}=\phi^{q+1} \lambda^{q+1} \tau^{q}=\lambda^{q+1} \phi^{n} \tau^{q}=\lambda^{q+1} \tau^{q} \phi^{q}$ $=\eta^{q} \phi^{q}$.

We want to use Theorem 2.5 to yield a canonical factorization of the set 
$G_{1} * \cdots * G_{n}$ as a Cartesian product. To do so we need the following elementary lemma.

LEMma 2.16. Let $1 \rightarrow K_{\stackrel{\lambda}{\lambda}}^{\rightarrow} \rightarrow \stackrel{\mu}{\rightarrow} H \rightarrow 1$ be a short exact sequence of groups and homomorphisms and let $\eta: H \rightarrow G$ be a transformation such that $\mu \eta=1$. Then we may define a $(1,1)$ correspondence $\sigma=\sigma(\lambda, \mu, \eta): K \times H \rightarrow G$ such that if the diagram

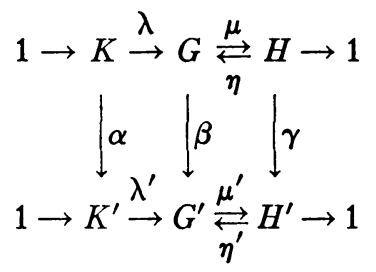

is commutative, $\mu^{\prime} \eta^{\prime}=1$, and $\alpha, \beta, \gamma$ are homomorphisms then the diagram

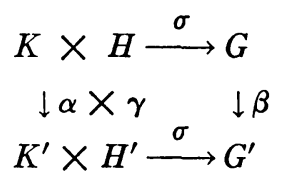

is commutative.

Proof. Suppressing the symbol for the map $\lambda$, we define $\sigma: K \times H \rightarrow G$ by $\sigma(k, h)=k \cdot \eta h$ and we define $\rho: G \rightarrow K \times H$ by $\rho(g)=\left(g \cdot(\eta \mu g)^{-1}, \mu g\right)$. Then

while

$$
\begin{aligned}
\sigma \rho(g) & =g \cdot(\eta \mu g)^{-1} \cdot \eta \mu g=g, \\
\rho \sigma(k, h) & =\left(k \cdot \eta h \cdot(\eta \mu(k \cdot \eta h))^{-1}, \mu(k \cdot \eta h)\right) .
\end{aligned}
$$

Now since $\mu$ is a homomorphism $\mu(k \cdot \eta h)=\mu k \cdot \mu \eta h=e \cdot h=h$. Thus

$$
\rho \sigma(k, h)=\left(k \cdot \eta h \cdot(\eta h)^{-1}, h\right)=(k, h),
$$

and $\sigma$ is a $(1,1)$ correspondence with inverse $\rho$. We prove naturality by observing that

$$
\beta \sigma(k, h)=\beta(k \cdot \eta h)=\beta k \cdot \beta \eta h=\alpha k \cdot \eta^{\prime} \gamma h=\sigma(\alpha k, \gamma h)=\sigma(\alpha \times \gamma)(k, h) .
$$

It remains now to study the kernel of $\kappa^{q}: G^{q+1} \rightarrow G^{q}$. It is clear that $g=\left\{g_{I}\right\} \in \operatorname{ker} \kappa^{q}$ if and only if $g_{I}=e$ when $\left(^{3}\right)|I|=q$. Thus ker $\kappa^{q}$ may be identified with $\times_{|I|=q+1}\left[G_{I}\right]$, where $\left[G_{I}\right]$ is the subgroup of $G_{I}$ given by

$$
\left[G_{I}\right]=\bigcap_{J \subset_{I}} \operatorname{ker} \pi_{J}^{I} .
$$

We digress temporarily to give another characterization of $\left[G_{I}\right]$. Let $i \in I$ and let $G_{i}^{N}$ be the normal closure of $G_{i}$ in $G_{I}$. Then

(3) That is, $\operatorname{ker} \kappa^{q}=F_{a}\left(G^{q+1}\right)$. 
Proposition 2.18

$$
\left[G_{I}\right]=\bigcap_{i \in I} G_{i}^{N}
$$

Proof. We show that $G_{i}^{N}=\operatorname{ker} \pi_{J}^{I}$ where $J=I-(i)$. It is clearly sufficient to take the case $I=\{1,2\}, i=1$, and we are then asserting that the kernel of the projection $G_{1} * G_{2} \rightarrow G_{2}$ is $G_{1}^{N}$. This fact is well-known and easily proved.

We now apply Lemma 2.16 to the sequence

$$
1 \rightarrow \operatorname{ker} \kappa^{q} \rightarrow G^{q+1} \underset{\eta^{q}}{\stackrel{\kappa^{q}}{\rightleftarrows}} G^{q} \rightarrow 1
$$

to prove

Proposition 2.19. There is a $(1,1)$ correspondence

$$
\sigma^{q}: \underset{|I|=q+1}{\times}\left[G_{I}\right] \times G^{q} \leftrightarrow G^{q+1}
$$

which is natural in the sense that the diagram

$$
\begin{aligned}
& \underset{|I|=q+1}{\times}\left[G_{I}\right] \times G^{q} \stackrel{\sigma^{q}}{\rightarrow} G^{q+1} \\
& \underset{|I|=q+1}{\times}\left[\phi_{I}\right] \mid \times \phi^{q} \rightarrow \downarrow \phi^{q+1} \\
& \underset{|I|=q+1}{\times}\left[H_{I}\right] \times H^{q} \stackrel{\sigma^{q}}{\rightarrow} H^{q+1}
\end{aligned}
$$

is commutative.

(Here we have introduced the obvious notation $\left[\phi_{I}\right]:\left[G_{I}\right] \rightarrow\left[H_{I}\right]$ for the homomorphism induced by the homomorphisms $\phi_{i}: G_{i} \rightarrow H_{i}$.)

By iteration we now obtain our main theorem.

TheORem 2.20. There is a $(1,1)$ correspondence

$$
\tau: \underset{I}{X}\left[G_{I}\right] \leftrightarrow *_{i} G_{i}
$$

which is natural in the sense that the diagram

$$
\begin{aligned}
& \underset{i}{\times}[G] \stackrel{\tau}{\rightarrow} *_{i} G_{i} \\
& \underset{I}{\times}\left[\phi_{I}\right]{ }_{I} \quad{ }^{\prime} \phi_{1} \\
& \underset{I}{\times}\left[H_{I}\right] \stackrel{\tau}{\rightarrow} *_{i} H_{i}
\end{aligned}
$$

is commutative. 
For we simply define $\tau$ by $\tau=\sigma^{n-1} \circ\left(1 \times \sigma^{n-2}\right) \circ \cdots \circ\left(1 \times \sigma^{2}\right) \circ\left(1 \times \sigma^{1}\right)$ where

$$
1 \times \sigma^{q}: \underset{|I|>q+1}{\times}\left[G_{I}\right] \times\left(\underset{|I|=q+1}{\times}\left[G_{I}\right] \times G^{q}\right) \rightarrow \underset{|I|>q+1}{\times}\left[G_{I}\right] \times G^{q+1} .
$$

However we may give an explicit description of the map $\tau$ and this we proceed to do. We have already ordered the $r$-strings for each $r$ and we extend the order to the set of all strings by declaring that $I$ precedes $J$ if $|I|>|J|$. Then given any string $I$ we have an embedding $i_{I}:\left[G_{I}\right] \rightarrow *_{i} G_{i}$ which is just the composition of the evident embeddings $\left[G_{I}\right] \rightarrow G_{I}, G_{I} \rightarrow *_{i} G_{i}$.

Theorem 2.21. The map $\tau: X_{I}\left[G_{I}\right] \rightarrow *_{i} G_{i}$, given by

$$
\tau\left\{g_{I}\right\}=\underset{I}{\operatorname{product}} i_{I}\left(g_{I}\right)
$$

satisfies the conditions of Theorem 2.20.

Proof. In fact we show that the map $\tau$ given by (2.22) is precisely the map we get from our definitions of $\eta^{q}$ and $\sigma^{q}$. We refer to an element of $\times_{I}\left[G_{I}\right]$ as an $n$-tuple

$$
\left\{g^{(n)}, \cdots, g^{(2)}, g^{(1)}\right\}
$$

where $g^{(q)} \in X_{|I|=q}\left[G_{I}\right]$; we may write $g^{(q)}=\left\{g_{I}\right\}_{|I|=q}$, so that $g_{I} \in\left[G_{I}\right]$.

We define a sequence of elements $h^{(q)} \in G^{q}$ by

$$
h^{(1)}=e, \quad h^{(q+1)}=\eta^{q}\left(g^{(q)} h^{(q)}\right), \quad 1 \leqq q \leqq n-1 .
$$

Notice that this definition makes sense because $g^{(q)} \in \operatorname{ker} \kappa^{q-1} \subseteq G^{q}$; notice also that

$$
\tau\left\{g_{I}\right\}=g^{(n)} h^{(n)},
$$

for we may show inductively that

$\left(1 \times \sigma^{q}\right) \circ \cdots \circ\left(1 \times \sigma^{1}\right)\left(g^{(n)}, \cdots, g^{(2)}, g^{(1)}\right)=\left(g^{(n)}, \cdots, g^{(q+2)}, g^{(q+1)} h^{(q+1)}\right)$.

Thus it remains to show that

$$
\underset{I}{\operatorname{product}} i_{I}\left(g_{I}\right)=g^{(n)} h^{(n)} .
$$

In fact we show more generally that

$$
\left(g^{(q)} h^{(q)}\right)_{I}=\underset{J \subseteq I}{\operatorname{product}} g_{J}
$$

where $G_{J}$ is regarded as embedded in $G_{I}$. Then $(2.24)_{n}$ is just (2.24) and (2.24) is trivial. Thus we assume $(2.24)_{q}$ and prove $(2.24)_{q+1}$.

Let us write $u=g^{(q)} h^{(q)}$ so that $u_{I}=$ product $_{J \subseteq I} g_{J}$. We prove by induction on $r$ that 


$$
\alpha_{r} \theta_{r}(u)_{I}=\underset{|J|>r+1 ; J \subseteq I}{\text { product }} g_{J}, \quad \beta_{r} \theta_{r}(u)=\underset{|J|=r+1}{\text { product }} g_{J} .
$$

Then $(2.25)_{0}$ is obvious (note that $u_{J}=g_{J}$ if $|J|=1$ ). Assuming (2.25) we find

$$
\begin{aligned}
\alpha_{r+1} \theta_{r+1}(u)_{I} & =\underset{\substack{|J|>r+1 ; J \subseteq I \\
\text { product }}}{ } g_{J}\left(\underset{|K|=r+2 ; K \subseteq I}{\text { product }} \underset{|L|>r+1 ; L \subseteq K}{\text { product }} g_{L}\right)^{-1} \\
& =\operatorname{product}_{|J|>r+1 ; J \subseteq I} g_{J}\left(\underset{|K|=r+2 ; K \subseteq I}{\text { product }} g_{K}\right)^{-1}=\underset{|J|>r+2 ; J \subseteq I}{\text { product }} g_{J},
\end{aligned}
$$

while $\beta_{r+1} \theta_{r+1}(u)=$ product $_{|K|=r+2} \operatorname{product}_{|L|>r+1 ; L \subseteq K} g_{L}=$ product $_{|K|=r+2} g_{K}$. This establishes $(2.25)_{r+1}$ and hence $(2.25)_{r}$ for each $r$. Thus

$$
\tau^{q}(u)=\prod_{s=q-1}^{0} \beta_{s} \theta_{s}(u)=\underset{|J| \leqq q}{\text { product }} g_{J} .
$$

Let $I$ be a $(q+1)$-string. Then

$$
\begin{aligned}
h_{I}^{(q+1)}=\left(\eta^{q} u\right)_{I} & =\left(\lambda^{q} \tau^{q} u\right)_{I}=\pi_{I} \underset{|J| \leqq q}{\operatorname{product}} g_{J} \\
& =\underset{J \subset I}{\text { product }} g_{J} \text { since } g_{J} \in\left[G_{J}\right] .
\end{aligned}
$$

Thus $\left(g^{(q+1)} h^{(q+1)}\right)_{I}=g_{I}$ product $\subset_{J} \subset_{I} g_{J}=$ product $_{J} \subseteq_{I} g_{J}$. Similarly $\left(g^{(q+1)} h^{(q+1)}\right)_{I}$ $=$ product $_{J \subseteq I} g_{J}$ if $|I|<q+1$ so that $(2.24)_{q+1}$ is proved and hence also the theorem.

We remark that this theorem has the important consequence that if, for any string $I_{0},\left[G_{I_{0}}\right]$ is embedded in $\times_{I}\left[G_{I}\right]$ by putting the remaining components equal to $e$ then $\tau \mid\left[G_{I_{0}}\right]$ is the inclusion and hence, in particular, a monomorphism. We also remark (less importantly!) that Theorem 2.21 could have been used to establish the naturality of $\tau$.

3. The groups $\left[G_{I}\right]$. In this section we examine the structure of the groups $\left[G_{I}\right]$. It is, of course sufficient to look at $\left[G_{I}\right]$ if $I=\{1, \cdots, n\}$, when we write $\left[G^{n}\right]$ for $\left[G_{I}\right]$. If $n=1$, then $\left[G_{1}\right]=G_{1}$ and there is nothing to be said.

THEOREM 3.1. If $n>1$, then $\left[G^{n}\right]$ is a free group. If moreover $G_{i}$ is a subgroup of $H_{i}, i=1, \cdots, n$, then $\left[G^{n}\right]$ is a free factor in $\left[H^{n}\right]$.

Proof. We first prove the theorem if $n=2$. Then $\left[G^{2}\right]=\left[G_{1}, G_{2}\right]$, the subgroup of $G_{1} * G_{2}$ freely generated by commutators $\left[g_{1}, g_{2}\right], g_{1} \in G_{1}, g_{2} \in G_{2}$, $g_{1} \neq e, g_{2} \neq e$. Thus $\left[G^{2}\right]$ is a free group and if $G_{i} \subseteq H_{i}, i=1,2$, then $\left[G^{2}\right]$ is a free factor in $\left[H^{2}\right]$.

Now assume $n>2$. Since $\left[G^{n}\right] \subseteq\left[G_{1}, G_{2} * \cdots * G_{n}\right]$ it follows from what we have proved that $\left[G^{n}\right]$ is a free group. Also since $\left[G^{n}\right]=\bigcap_{|I|=n-1} \operatorname{ker} \pi_{I}$ it is clear that 


$$
\left[G^{n}\right]=*_{i} G_{i} \cap\left[H^{n}\right] .
$$

Hence, since $\left[G^{n}\right] \subseteq\left[G_{1}, G_{2} * \ldots * G_{n}\right]$ we have

$$
\left[G^{n}\right]=\left[G_{1}, G_{2} * \cdots * G_{n}\right] \cap\left[H^{n}\right] .
$$

Our theorem is proved $\left({ }^{4}\right)$ when we establish

Lemma 3.3. If $A$ is a free factor in $B$ and $C$ is a subgroup of $B$ then $A \cap C$ is a free factor in $C$.

For we put $B=\left[H_{1}, H_{2} * \cdots * H_{n}\right], A=\left[G_{1}, G_{2} * \cdots * G_{n}\right], C=\left[H^{n}\right]$. The proof of the lemma applies the remark on p. 269 of [4] on the proof of the Kurosh subgroup theorem. Thus the subgroup theorem asserts that if a group $M$ is expressed as a free product,

$$
M=*_{i} M_{i}
$$

and if $N \subseteq M$ then

$$
N=F * *_{j} N_{j},
$$

where $F$ is free and each $N_{j}$ is conjugate in $M$ to a subgroup of some $M_{i}$. The remark is that if $m \in M$ and if $D=m^{-1} M_{i} m \cap N \neq(e)$, then the decomposition of $N$ which arose in the proof of the theorem had the property that $D$ is conjugate in $N$ to some $N_{j}$.

We are given $B=A * S$ for some $S$. Then either $A \cap C=(e)$ or we may write $C=R * T$ where $R$ is conjugate in $C$ to $A \cap C$. This shows that $A \cap C$ is a free factor in $C$ and the lemma is proved.

TheOREM 3.4. If $\phi_{i}: G_{i} \rightarrow H_{i}, i=1, \cdots, n$, are homomorphisms inducing $\left[\phi^{n}\right]:\left[G^{n}\right] \rightarrow\left[H^{n}\right]$ and if $M_{i}=\phi_{i} G_{i}$ then

$$
\left[\phi^{n}\right]\left[G^{n}\right]=\left[M^{n}\right] .
$$

Proof. It is plain that $\left[\phi^{n}\right]\left[G^{n}\right] \subseteq\left[H^{n}\right]$ and $\left[\phi^{n}\right]\left[G^{n}\right] \subseteq *_{i} M_{i}$. Thus

$$
\left[\phi^{n}\right]\left[G^{n}\right] \subseteq\left[M^{n}\right] .
$$

Now let $m \in\left[M^{n}\right]$; then certainly $m=\left(*_{i} \phi_{i}\right)(g)$ for some $g \in *_{i} G_{i}$ and we wish to show that we may choose $g$ in $\left[G^{n}\right]$. We filter $*_{i} G_{i}=G^{n}$ by declaring $g \in F_{s}\left(G^{n}\right)$ if $\pi_{I}(g)=e$ whenever $\left(^{5}\right)|I|=s$. We then deduce the theorem from the following assertion.

Proposition 3.5. If $g \in F_{s-1}\left(G^{n}\right), s<n$, and $\phi^{n} g \in\left[M^{n}\right]$ then $g=g^{\prime} g^{\prime \prime}$ with $g^{\prime} \in F_{s}\left(G^{n}\right), \phi^{n}\left(g^{\prime \prime}\right)=e$.

To prove 3.5 , consider $g^{\prime \prime}=\operatorname{product}_{|J|=s} \pi_{J} g$, where, as usual, $G_{J}$ is re-

(4) The author is indebted to Graham Higman for the lemma and its proof.

(5) This is essentially the same filtration as that used in the proof of Theorem 2.5. 
garded as embedded in $G^{n}$. Then $\phi^{n}\left(g^{\prime \prime}\right)=\phi^{n} \quad$ product $_{|J|=s} \pi_{J} g$ ) $=\operatorname{product}_{|J|=s} \pi_{J} \phi^{n} g=e$, because $\phi^{n} g \in\left[M^{n}\right]$ and $s<n$. Also, if $g^{\prime}=g g^{\prime \prime-1}$ and $K$ is an $s$-string, then $\pi_{K} g^{\prime}=\pi_{K} g$ (product $\left.|J|=s \pi_{K} \pi_{J} g\right)^{-1}$. Now (compare 2.10)

$$
\begin{aligned}
\pi_{K} \pi_{J} g & =\pi_{J} g \text { if } K=J, \\
& =e \text { if } K \neq J \text { since } g \in F_{s-1} .
\end{aligned}
$$

Thus $\pi_{K} g^{\prime}=\pi_{K} g\left(\pi_{K} g\right)^{-1}=e$ so that $g^{\prime} \in F_{s}\left(G^{n}\right)$ and the proposition is proved. Since $F_{0}\left(G^{n}\right)=G^{n}$ and $F_{n-1}\left(G^{n}\right)=\left[G^{n}\right]$, we complete the proof of the theorem by iterated application of the proposition.

CoRollary 3.6. If $n>1$ then $\left[\phi^{n}\right]$ maps $\left[G^{n}\right]$ onto a free factor in $\left[H^{n}\right]$.

4. Group complexes. We indicate here briefly the implication of the foregoing results in the category of group complexes. The main applications in homotopy theory will be described elsewhere.

We recall that a group complex $\left({ }^{6}\right)$ is a c.s.s. complex whose sections are groups and whose face and degeneracy operators are homomorphisms. Moreover a map of group complexes is a c.s.s. map which is a homomorphism of each section. Given group complexes $G_{1}, \cdots, G_{n}$ we may form in an obvious way their free product $G_{1} * \cdots * G_{n}$ and their direct product $G_{1} \times \cdots \times G_{n}$ and the natural map $\kappa$ from $G_{1} * \cdots * G_{n}$ to $G_{1} \times \cdots \times G_{n}$ is then a map of group complexes. The theory of $\$ 2$ may then be applied to this situation and we may prove the following obvious extension of Theorems 2.20 and 2.21.

THEOREM 4.1. $\left[G_{I}\right]$ is a subgroup complex of $G_{I}$. Moreover there is a c.s.s. equivalence

$$
\tau: \underset{I}{X}\left[G_{I}\right] \leftrightarrow *_{i} G_{i}
$$

which is natural in the sense that the diagram

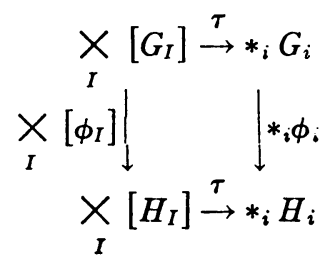

is commutative, where $\phi_{i}: G_{i} \rightarrow H_{i}, i=1, \cdots, n$ are maps of group complexes. Indeed, $\tau$ may be defined by

$$
\tau\left\{g_{I}\right\}=\underset{I}{\operatorname{product}} i_{I}\left(g_{I}\right) .
$$

It is only necessary to observe that the fact that $\tau$ is here a c.s.s. map and (6) Or c.s.s. group; see $[2 ; 3]$. 
the fact that it is natural are both consequences of the naturality of $\tau$ in Theorem 2.20.

In [2] Kan describes a functor $G$ passing from the category of reduced c.s.s. complexes (complexes with a single vertex) to the category of group complexes. Let $K_{1} \vee \cdots \vee K_{n}$ be the reduced c.s.s. complex obtained from the disjoint union of the reduced complexes $K_{i}$ by identifying their vertices (and the degeneracies of the vertices). Then it is clear that

$$
G\left(K_{1} \vee \cdots \vee K_{n}\right)=G K_{1} * \cdots * G K_{n} .
$$

Thus there is a c.s.s. equivalence

$$
\tau: \underset{I}{\times}\left[G(K)_{I}\right] \rightarrow G\left(K_{1} \vee \cdots \vee K_{n}\right)
$$

which, restricted to any $G(K)_{I}$, is a group complex monomorphism. This assertion takes on a topological significance when the function $G$ is interpreted as the loop-functor.

The results of $\S 3$ enable us to conclude that, in the group complex $\left[G_{I}\right]$, $|I|>1$, the face and degeneracy operators are always onto free factors; and that if $\phi_{i}: G_{i} \rightarrow H_{i}$ are maps of group complexes then $\left[\phi_{I}\right]$ maps $\left[G_{I}\right]$ onto a free factor in $\left[H_{I}\right]$. Moreover, $\left[G_{I}\right],|I|>1$, is free in each dimension.

It seems reasonable to conjecture that, in fact, a stronger theorem than Theorem 3.1 holds; namely, that a set $S$ of free generators of the group $\left[H^{n}\right], n>1$, may be chosen so that, for any system of subgroups $G_{i}$ of $H_{i}$, $i=1, \cdots, n$, the elements of $S \cap\left[G^{n}\right]$ constitute a set of generators $\left({ }^{7}\right)$ of $\left[G^{n}\right]$. This would imply that the group complexes $\left[G_{I}\right],|I|>1$, of this section are free in the sense of Kan [3].

\section{REFERENCES}

1. R. H. Fox, On Lusternik-Schnirelmann category, Ann. of Math. vol. 42 (1941) p. 333. p. 282.

2. D. M. Kan, A combinatorial definition of homotopy groups, Ann. of Math. vol. 67 (1958)

3. - On homotopy theory and c.s.s. groups, Ann. of Math. vol. 68 (1958) p. 38.

4. A. G. Kurosch, Gruppentheorie, Berlin, Akademie Verlag, 1953.

CORNell University, ITHACA, NEW YORK

(7) This conjecture has been proved by D. E. Cohen (see a forthcoming publication). 